\title{
Patterns of health care utilization related to initiation of amitriptyline, duloxetine, gabapentin, or pregabalin in fibromyalgia
}

\author{
Seoyoung C Kim*, Joan E Landon ${ }^{1}$ and Yvonne C Lee ${ }^{2}$
}

\begin{abstract}
Introduction: Several pharmacologic treatments are available for fibromyalgia, but little is known about the comparative effectiveness of these treatments on health care utilization.

Methods: Using US commercial insurance claims data (covering 2007 to 2009), we conducted a cohort study to examine the comparative effectiveness of amitriptyline, duloxetine, gabapentin, and pregabalin on health care utilization in patients with fibromyalgia. We measured patients' medication adherence using the proportion of days covered (PDC) and estimated multivariable rate ratios (RRs) for outpatient visits, prescriptions, hospitalization, and emergency department (ED) visits in propensity score (PS)-matched cohorts.

Results: Cohorts of 8,269 amitriptyline, 9,941 duloxetine, and 18,613 gabapentin initiators were compared with their PS-matched pregabalin initiators. During the baseline 180-day period, patients had, on average, seven to nine physician visits, including six to eight specialist visits, and received eight prescription drugs. The mean PDC up to 180 days varied from $38.6 \%$ to $67.7 \%$. The number of outpatient visits, prescriptions, and hospitalizations decreased slightly after initiating one of the study drugs, but the number of ED visits increased after treatment initiation. Compared to pregabalin, duloxetine was associated with decreased outpatient visits (RR, 0.94; 95\% confidence interval (Cl), 0.88 to 1.00), prescriptions ( $R R, 0.94 ; 95 \% \mathrm{Cl}, 0.90$ to 0.98 ), hospitalizations ( $R R, 0.75 ; 95 \% \mathrm{Cl}, 0.68$ to 0.83 ), and $\mathrm{ED}$ visits (RR, $0.85 ; 95 \% \mathrm{Cl}, 0.79$ to 0.91 ). Little difference in health care utilization rates was noted among amitriptyline and gabapentin initiators compared to those who were started on pregabalin.

Conclusions: Fibromyalgia patients had high health care utilization before and after initiation of amitriptyline, duloxetine, gabapentin, or pregabalin. Medication adherence was suboptimal. Overall, fibromyalgia treatment had little impact on reducing health care utilization, but duloxetine initiators had less health care utilization than those started on pregabalin.
\end{abstract}

\section{Introduction}

Fibromyalgia is a common, highly disabling syndrome characterized by chronic widespread pain, fatigue, and problems with sleep, memory, and mood [1-3]. Treatment of fibromyalgia is challenging and often requires both pharmacologic and nonpharmacologic treatments $[4,5]$. For pharmacologic treatment, tricyclic antidepressants (TCAs) such as amitriptyline, selective serotonin reuptake inhibitors (SSRIs), serotonin and noradrenaline

\footnotetext{
* Correspondence: skim62@partners.org

1 Division of Pharmacoepidemiology and Pharmacoeconomics, Brigham and Women's Hospital and Harvard Medical School, 1620 Tremont St, Suite 3030, Boston, MA 02120, USA

Full list of author information is available at the end of the article
}

reuptake inhibitors (SNRIs) such as duloxetine and milnacipran, and monoamine oxidase inhibitors are used to reduce pain, depression, fatigue, and health-related quality of life [6,7]. Gabapentin and pregabalin are also commonly prescribed to ameliorate pain and sleep problems associated with fibromyalgia [6,7].

Patients with fibromyalgia are known to be heavy users of health care systems, including outpatient visits, hospitalizations, and prescription drug use [8-10]. Despite the number of available drugs for fibromyalgia, few studies have directly compared the effects of different pharmacologic agents for fibromyalgia on health care utilization. In studies comparing pre- and posttreatment, pregabalin initiators had increased inpatient and outpatient visits 
after initiating pregabalin, whereas duloxetine initiators had decreased inpatient and outpatient visits after initiating duloxetine [11-13]. In another study, researchers reported increases in total health care costs after initiating treatment with pregabalin or duloxetine for fibromyalgia [10]. In a study comparing pregabalin to TCAs, pregabalin initiators had increased total health care costs from pretreatment to follow-up [14]. Although all of these studies were performed using US claims data, the effects of fibromyalgia treatment on health care utilization may vary across different health care systems, particularly across different countries.

The objective of this study was to examine the comparative effectiveness of four commonly used drugs for fibromyalgia-amitriptyline, duloxetine, gabapentin, and pregabalin-on the rate of health care utilization in a US population-based cohort of commercially insured patients with fibromyalgia. Patients' adherence to these drug regimens (that is, continuation or discontinuation) was also assessed. We hypothesized that the effect of amitriptyline, duloxetine, or gabapentin on health care utilization would differ from the effect of pregabalin.

\section{Methods}

\section{Data source}

We conducted a cohort study using claims data obtained from UnitedHealthcare, a US commercial health insurance plan, which insures primarily working adults and their family members, for the period from 1 January 2007 to 31 December 2009. The details of the data source are presented elsewhere [15]. The study protocol was approved by the Institutional Review Board of the Brigham and Women's Hospital. Patients' informed consent was not required by the Institutional Review Board, as the dataset was deidentified to protect subject confidentiality.

\section{Study cohort}

Among patients ages 18 years and older who had at least one visit coded with the International Classification of Diseases, Ninth Revision, Clinical Modification (ICD 9-CM), code $729.1 x$, for fibromyalgia, new users of a study drug-amitriptyline, duloxetine, gabapentin, or pregabalin -were identified. Milnacipran users were initially considered, but they were subsequently excluded because of the small number of patients taking milnacipran (less than $0.5 \%$ of all subjects). To be included in the study cohort, we required patients to have at least 180 days of continuous health plan eligibility before receiving the first prescription of a study drug. Patients were required to be naïve to all four drugs during the 180-day baseline period. We excluded patients who were prescribed more than one study drug at the same index date.

Follow-up time started on the index date, defined as the dispensing date of the first study drug, and ended at the first of any of the following censoring events: discontinuation or switching of study drugs, loss of health plan eligibility, end of study database, or death.

\section{Health care utilization}

Changes in health care utilization factors (for example, the number of outpatient visits to any physicians, primary care physicians and any specialists; number of prescription drugs; acute care hospitalizations; emergency department (ED) visits; physical therapy) were assessed during follow-up.

\section{Covariates}

Variables potentially related to fibromyalgia symptoms, initiation of the study drugs, or health care utilization were assessed using data from the 180-day baseline period. These variables included age, sex, fibromyalgia-related comorbidities (back pain, chronic headache, depression, anxiety, chronic pain, fatigue, sleep disorder, and abdominal pain condition), other comorbidities (hypertension, diabetes, stroke, cardiovascular disease, malignancy, and inflammatory arthritis), use of other medications (opioids, benzodiazepines, anticonvulsants excluding gabapentin and pregabalin, TCAs excluding amitriptyline, SSRIs, SNRIs excluding duloxetine, other antidepressants, muscle relaxants, nonbenzodiazepine sleep disorder drugs, oral glucocorticoids, nonsteroidal anti-inflammatory drugs (NSAIDs), topical analgesics, migraine drugs, and gastrointestinal protective drugs), and health care utilization factors (number of outpatient visits to any physicians, primary care physicians, and specialists; number of prescription drugs; physical therapy; ED visits; and acute hospitalizations) $[15,16]$. Additional files 1 and 2 contain the lists of diagnoses and procedure codes and medications. To quantify patients' comorbidities, we also calculated the Deyo-adapted Charlson Comorbidity Index based on the ICD-9-CM $[17,18]$.

\section{Statistical analysis}

We compared the baseline characteristics among the initiators of each study drug. To control for potential confounding by indication, we used propensity score (PS) estimation [19]. In multivariable logistic regression models using pregabalin initiators as the common reference, we estimated a PS. Pregabalin was chosen as the common reference because (1) we wanted to directly compare one treatment approved by the US Food and Drug Administration (FDA) to another FDA-approved treatment (duloxetine) and other non-FDA-approved treatments (amitriptyline and gabapentin), and (2) the number of pregabalin initiators was greater than that of duloxetine initiators. All the aforementioned baseline covariates except health care utilization factors were included in the logistic models. Each pair-amitriptyline 
versus pregabalin, duloxetine versus pregabalin, and gabapentin versus pregabalin-was then matched on the PS using the greedy matching algorithm [20]. To measure patients' adherence to their fibromyalgia treatments, we calculated the proportion of days covered (PDC) as the total number of days' supply provided by prescriptions for a given study drug up to 180 days from the index date divided by 180 days.

Adjusting for baseline health care utilization factors, we used multivariable Cox models to estimate rate ratios (RR) for ED visits, acute care hospitalizations, and physical therapy during the follow-up period in all three PSmatched pairs [21]. Multivariable Poisson regression, adjusted for baseline health care utilization factors, was used to estimate RR for outpatient visits to any physician, primary care physicians, and any specialists, as well as the number of prescription drugs, during the follow-up period in all three PS-matched pairs. To deal with the potential overdispersion in Poisson models, the standard errors were adjusted with the scale option in SAS 9.3 software (SAS Institute, Cary, NC, USA). To assess a long-term effect of treatment, a subgroup analysis using multivariable Poisson and Cox regression models was performed in patients with treatment duration of at least 180 days. All analyses were done using SAS 9.3 software.

\section{Results}

\section{Study cohort}

Among 116,183 patients who had at least one diagnosis of fibromyalgia between 2007 and 2009, we identified 13,404 amitriptyline, 18,420 duloxetine, 23,268 gabapentin, and 19,286 pregabalin initiators [15]. Of those, 8,269 amitriptyline, 9,941 duloxetine, and 18,613 gabapentin initiators were matched to pregabalin initiators on their PS with a ratio of 1:1. Thus, different subsets of pregabalin users were compared for each pairwise analysis.

\section{Baseline characteristics}

Demographic characteristics, comorbidities, and medications at baseline were well balanced and statistically not different between the PS-matched cohorts (Table 1). The mean age ranged from 47.7 to 50.0 years, depending on the treatment pairs. The majority of patients were women (77\% to $82 \%)$. The median starting daily doses were $25 \mathrm{mg}$ for amitriptyline, $60 \mathrm{mg}$ for duloxetine, $300 \mathrm{mg}$ for gabapentin, and $75 \mathrm{mg}$ for pregabalin. Back pain was the most common comorbidity (52\% to $63 \%$ ), followed by hypertension (30\% to 36\%). During the 180 day baseline, more than one-half of patients received at least one prescription for opioids and one-third used benzodiazepines. Use of SSRIs, non-SSRIs/SNRIs antidepressants, muscle relaxants, oral steroids, and NSAIDs was also common.
On average, patients had 7.4 to 8.8 outpatient visits and 5.9 to 7.7 specialist visits during the 180 -day baseline period. The mean number of prescription drugs at baseline was also high, between 7.9 and 8.4. Approximately $10 \%$ to $12 \%$ of patients had at least one ED visit and $10 \%$ to $14 \%$ had at least one acute hospitalizations during the 180-day baseline period.

\section{Continuation of fibromyalgia medication}

The mean (standard deviation (SD)) duration of treatment was 77 (92) days for the amitriptyline-pregabalin pairs, 94 (110) days for the duloxetine-pregabalin pairs, and 82 (97) days for the gabapentin-pregabalin pairs. The mean (SD) PDCs up to 180 days of follow-up for the amitriptyline and pregabalin pair were $38.6 \%$ (30.1) and $50.0 \%$ (31.2), respectively. Among the amitriptyline and pregabalin initiators, $7.9 \%$ remained on the treatment for at least 180 days. For the duloxetine and pregabalin pair, the mean (SD) PDC values were 55.0\% (34.0) and $67.8 \%(33.4)$, respectively. Among duloxetine and pregabalin initiators, $16.5 \%$ and $8.3 \%$, respectively, remained on the treatment for at least 180 days. The mean PDCs (SD) up to 180 days of follow-up were $45.0 \%$ (32.5) for gabapentin initiators and 55.4\% (32.4) for pregabalin. Among gabapentin and pregabalin initiators, $9.0 \%$ and $9.9 \%$, respectively, remained on the treatment for at least 180 days (Table 2). Of the patients who remained on the treatment for 180 days, the dose was increased in only $5.7 \%$ of amitriptyline, $8.7 \%$ of duloxetine and gabapentin, and $13.9 \%$ of pregabalin initiators.

\section{Changes in health care utilization rates}

The mean number of outpatient visits and the proportion of patients with at least one hospitalization numerically decreased slightly after treatment initiation compared to before treatment initiation. The number of prescription drugs did not change, and the proportion of patients with at least one ED visit or at least one physical therapy visit increased after treatment initiation in all groups. In the amitriptyline and pregabalin pair, $4.2 \%$ of patients taking amitriptyline and $4.5 \%$ of patients taking pregabalin were started on another study drug during follow-up. Similarly, $5.6 \%$ of duloxetine and $7.1 \%$ of pregabalin initiators were prescribed another study drug during follow-up. In the gabapentin-pregabalin pair, 11.5\% of gabapentin initiators and $13.8 \%$ of pregabalin initiators received another study drug during follow-up. Table 3 shows patients' unadjusted health care utilization patterns before and after treatment initiation.

In multivariable Poisson and Cox regression analyses (Table 4), duloxetine initiation was associated with significantly decreased outpatient visits (RR, 0.94; 95\% CI, 0.88 to 1.00 ), prescription drug use (RR, $0.94 ; 95 \% \mathrm{CI}$, 0.90 to 0.86 ), hospitalizations ( $\mathrm{RR}, 0.75$; $95 \% \mathrm{CI}, 0.68$ to 
Table 1 Baseline characteristics of the propensity score-matched cohorts in the 180 days prior to initiation of amitriptyline, duloxetine, gabapentin, or pregabalin ${ }^{a}$

\begin{tabular}{|c|c|c|c|c|c|c|}
\hline & Amitriptyline & Pregabalin & Duloxetine & Pregabalin & Gabapentin & Pregabalin \\
\hline Number of patients & 8,269 & 8,269 & 9,941 & 9,941 & 18,613 & 18,613 \\
\hline \multicolumn{7}{|l|}{ Demographics } \\
\hline Age (yr) & $47.7 \pm 12.4$ & $47.7 \pm 11.7$ & $48.3 \pm 11.6$ & $48.2 \pm 11.4$ & $50.0 \pm 12.6$ & $50.0 \pm 11.8$ \\
\hline Female & 79.6 & 79.8 & 82.0 & 82.0 & 77.3 & 77.6 \\
\hline \multicolumn{7}{|l|}{ Comorbidities } \\
\hline Back pain & 52.4 & 52.5 & 55.3 & 55.7 & 63.2 & 63.3 \\
\hline Headache & 26.0 & 26.3 & 22.2 & 22.6 & 21.6 & 21.7 \\
\hline Depression & 10.6 & 10.5 & 16.3 & 16.7 & 12.0 & 12.1 \\
\hline Anxiety & 12.6 & 12.7 & 15.9 & 16.6 & 13.0 & 13.0 \\
\hline Abdominal pain & 4.4 & 4.3 & 4.2 & 4.0 & 3.5 & 3.6 \\
\hline Neuropathic pain & $25.4^{*}$ & $33.4^{*}$ & $30.4^{*}$ & $36.1^{*}$ & $41.6^{*}$ & $40.5^{*}$ \\
\hline Sleep disorder & 16.7 & 16.2 & 18.3 & 18.4 & 16.5 & 16.5 \\
\hline Diabetes & 11.5 & 11.3 & 12.7 & 12.8 & 15.9 & 15.8 \\
\hline Hypertension & 29.8 & 30.0 & 31.6 & 31.2 & 35.7 & 35.7 \\
\hline Cardiovascular disease & 2.0 & 2.0 & 2.0 & 2.1 & 3.0 & 2.9 \\
\hline Stroke & 3.2 & 3.1 & 3.2 & 3.0 & 4.0 & 3.8 \\
\hline Inflammatory arthritis & 10.8 & 10.7 & 12.9 & 13.2 & 13.6 & 13.9 \\
\hline Malignancy & 4.5 & 4.6 & 4.8 & 4.6 & 5.9 & 5.8 \\
\hline Comorbidity index score $^{b}$ & $0.5 \pm 1.0$ & $0.5 \pm 1.0$ & $0.6 \pm 1.0$ & $0.6 \pm 1.0$ & $0.7 \pm 1.2$ & $0.7 \pm 1.2$ \\
\hline
\end{tabular}

Medications

\begin{tabular}{|c|c|c|c|c|c|c|}
\hline Opioids & 55.3 & 56.1 & 59.8 & 59.5 & 68.2 & 68.5 \\
\hline $\mathrm{TCAs}^{\mathrm{c}}$ & 10.9 & 10.4 & 3.9 & 3.9 & 4.8 & 4.7 \\
\hline SSRIS & 18.4 & 17.7 & 22.1 & 22.6 & 21.4 & 21.6 \\
\hline SNRIs ${ }^{d}$ & 5.4 & 5.7 & 17.8 & 17.1 & 8.6 & 8.7 \\
\hline Anticonvulsants $^{\mathrm{e}}$ & 12.5 & 11.8 & 17.5 & 17.3 & 39.0 & 39.0 \\
\hline Other antidepressants & 11.4 & 11.7 & 17.1 & 17.3 & 15.4 & 15.6 \\
\hline BZDs & 29.9 & 29.7 & 36.5 & 36.6 & 35.2 & 35.2 \\
\hline Sleeping disorder drugs ${ }^{f}$ & 19.9 & 20.2 & 23.3 & 22.6 & 21.3 & 21.9 \\
\hline Migraine drugs & 8.3 & 8.7 & 7.4 & 7.7 & 6.9 & 6.9 \\
\hline Muscle relaxants & 31.1 & 31.6 & 33.2 & 33.2 & 39.3 & 39.6 \\
\hline Topical analgesics & 3.5 & 3.6 & 4.5 & 4.7 & 6.7 & 6.8 \\
\hline Oral steroids & 26.5 & 26.2 & 26.3 & 26.3 & 31.0 & 31.3 \\
\hline NSAIDs & 33.6 & 33.4 & 35.0 & 34.9 & 40.4 & 40.8 \\
\hline Gl protective drugs & 21.9 & 21.9 & 22.6 & 22.5 & 25.2 & 25.4 \\
\hline \multicolumn{7}{|l|}{ h care utilization ${ }^{9}$} \\
\hline Outpatient visits, $n$ & $7.4 \pm 6.9^{*}$ & $7.7 \pm 7.3$ & $8.3 \pm 7.5$ & $8.2 \pm 8.2$ & $8.8 \pm 8.4^{*}$ & $8.6 \pm 8.1$ \\
\hline PCP visits, $n$ & $3.6 \pm 3.6$ & $3.5 \pm 3.5$ & $3.8 \pm 3.5$ & $3.7 \pm 3.7$ & $3.9 \pm 4.2^{*}$ & $3.8 \pm 3.7$ \\
\hline Specialist visits, $n$ & $5.9 \pm 7.3^{*}$ & $6.4 \pm 8.0$ & $6.5 \pm 7.9^{*}$ & $6.8 \pm 9.4$ & $7.6 \pm 8.8$ & $7.7 \pm 9.5$ \\
\hline Prescription drugs, $n$ & $7.9 \pm 5.4$ & $8.0 \pm 5.2$ & $8.6 \pm 5.4$ & $8.6 \pm 5.4$ & $9.4 \pm 5.7^{*}$ & $9.2 \pm 5.6$ \\
\hline Acute hospitalizations, $n$ & $9.8^{*}$ & 10.9 & $9.9^{*}$ & 11.2 & $14.2^{*}$ & 13.3 \\
\hline ED visits, $n$ & $11.8^{*}$ & 10.5 & 9.9 & 10.3 & $12.4^{*}$ & 10.9 \\
\hline
\end{tabular}

${ }^{a} B Z D s$, Benzodiazepines; ED, Emergency department; GI, Gastrointestinal; NSAIDs, Nonsteroidal anti-inflammatory drugs; PCP, Primary care physician; SNRI, Serotonin and norepinephrine reuptake inhibitor; SSRI, Selective serotonin reuptake inhibitor; TCA, Tricyclic antidepressant. Data are expressed as percentage or mean \pm SD. ${ }^{b}$ Deyo-adapted Charlson Comorbidity Index $[17,18]$. ${ }^{\mathrm{C}}$ All TCAs except amitriptyline. ${ }^{\mathrm{d}}$ All SNRIs except duloxetine. ${ }^{\mathrm{e}} \mathrm{All}$ anticonvulsants except gabapentin and pregabalin. ${ }^{f}$ Nonbenzodiazepine drugs only. ${ }^{9}$ Not included in the PS model. *Statistically significant difference compared to the reference group $(P<0.05)$. 
Table 2 Patients' continuation of fibromyalgia treatment ${ }^{a}$

\begin{tabular}{|c|c|c|c|c|c|c|}
\hline & Amitriptyline & Pregabalin & Duloxetine & Pregabalin & Gabapentin & Pregabalin \\
\hline Number of patients & 8,269 & 8,269 & 9,941 & 9,941 & 18,613 & 18,613 \\
\hline \multicolumn{7}{|l|}{ Treatment duration } \\
\hline 0 to 90 days & 80.9 & 79.7 & 63.5 & 78.6 & 78.1 & 75.7 \\
\hline 91 to 180 days & 11.2 & 12.4 & 20.0 & 13.0 & 13.0 & 14.3 \\
\hline 181 to 270 days & 3.4 & 4.1 & 7.6 & 4.2 & 4.2 & 4.9 \\
\hline 271 to 365 days & 2.1 & 1.7 & 4.0 & 1.7 & 2.1 & 2.2 \\
\hline Over 365 days & 2.4 & 1.2 & 4.9 & 2.5 & 2.7 & 2.8 \\
\hline PDC up to 180 days & $38.6 \pm 30.1$ & $50.0 \pm 31.2$ & $55.0 \pm 34.0$ & $67.8 \pm 33.4$ & $45.0 \pm 32.5$ & $55.4 \pm 32.4$ \\
\hline
\end{tabular}

${ }^{\mathrm{a} P D C}$, Proportion of days covered. Data are percentage or mean \pm SD.

0.83), ED visits ( $R R, 0.85 ; 95 \% \mathrm{CI}, 0.79$ to 0.91 ), and physical therapy visits (RR, $0.81 ; 95 \% \mathrm{CI}, 0.71$ to 0.92 ), compared to pregabalin. Amitriptyline initiators had similar rates of health care utilization, except for the significantly lower rate of hospitalization (RR, 0.70; 95\% CI, 0.62 to 0.80 ) and outpatient visits (RR, $0.91 ; 95 \% \mathrm{CI}$, 0.84 to 0.99 ), compared to pregabalin. Little difference in the rate of health care utilization existed between gabapentin and pregabalin initiators.

In a subgroup of patients who received at least 180 days of treatment, amitriptyline, duloxetine, and gabapentin initiators had lower rates of outpatient visits and specialist visits compared to pregabalin initiators (Table 5).

\section{Discussion}

In this large, US population-based cohort study, patients newly prescribed amitriptyline, duloxetine, gabapentin, or pregabalin for fibromyalgia generally had high health care utilization before and after treatment initiation. None of the four fibromyalgia treatments seemed to have much impact on health care utilization; however, duloxetine initiators had significantly fewer outpatient and ED visits and hospitalizations, less prescription drug use, and fewer physical therapy appointments compared to pregabalin initiators. As reported in prior research, depression is a predictor of high health care use [22]. The beneficial effect of duloxetine versus pregabalin seen in this study may be attributable to the fact that duloxetine is also an antidepressant and thus manages depressive mood in patients with fibromyalgia. Amitriptyline initiators also had fewer hospitalizations and physical therapy visits during follow-up than pregabalin initiators did.

Our study highlights several important issues in the management of fibromyalgia. First, fibromyalgia drugs appear to have little effect on reducing health care utilization by patients with fibromyalgia, as seen in previous studies of pregabalin, duloxetine, and TCAs [10-13]. Whether the lack of effect on health care utilization is due to inadequate benefits or concurrent benefit with new side effects of treatment is unclear. It is also difficult to determine whether the use of health care was inappropriately high or clinically necessary. Second, discontinuation rates are high across all study drugs. In previous studies, researchers have found that the effectiveness of these medications is limited [23], and many patients discontinue these medications due to side effects [24]. Third, pharmacologic treatment alone may not be effective in fibromyalgia [25]. Prior meta-analyses of various nonpharmacologic treatments such as massage therapy, aquatic physical therapy, balneotherapy, and hydrotherapy showed mixed results on fibromyalgia symptoms [25-28], although beneficial effects of aerobic exercise and cognitive behavioral therapy on pain and mood were consistently noted $[29,30]$. Nonetheless, in future longitudinal study, investigators should explore the utility of multifaceted approaches to the treatment of

Table 3 Crude changes in health care utilization pre- and post-index date among propensity score-matched cohorts ${ }^{a}$

\begin{tabular}{|c|c|c|c|c|c|c|c|c|c|c|c|c|}
\hline & \multicolumn{2}{|c|}{ Amitriptyline } & \multicolumn{2}{|c|}{ Pregabalin } & \multicolumn{2}{|c|}{ Duloxetine } & \multicolumn{2}{|c|}{ Pregabalin } & \multicolumn{2}{|c|}{ Gabapentin } & \multicolumn{2}{|c|}{ Pregabalin } \\
\hline & Pre & Post & Pre & Post & Pre & Post & Pre & Post & Pre & Post & Pre & Post \\
\hline Mean number of outpatient visits & 7.4 & 5.5 & 7.7 & 6.2 & 8.3 & 6.3 & 8.2 & 6.5 & 8.8 & 6.9 & 8.6 & 7.0 \\
\hline Mean number of PCP visits & 3.6 & 2.8 & 3.5 & 2.8 & 3.8 & 2.8 & 3.7 & 2.9 & 3.9 & 3.1 & 3.8 & 3.1 \\
\hline Mean number of specialist visits & 5.9 & 4.3 & 6.4 & 5.0 & 6.5 & 4.7 & 6.8 & 5.2 & 7.6 & 5.8 & 7.7 & 5.9 \\
\hline Mean number of prescription drugs & 7.9 & 7.9 & 8.0 & 8.3 & 8.6 & 8.4 & 8.6 & 8.9 & 9.4 & 9.3 & 9.2 & 9.5 \\
\hline Any acute hospitalization (\%) & 9.8 & 5.6 & 10.9 & 7.9 & 9.9 & 7.7 & 11.2 & 8.4 & 14.2 & 9.7 & 13.3 & 10.5 \\
\hline Any ED visit (\%) & 11.8 & 12.4 & 10.5 & 13.4 & 9.9 & 15.5 & 10.3 & 14.5 & 12.4 & 17.0 & 10.9 & 16.9 \\
\hline Physical therapy (\%) & 4.4 & 7.4 & 5.1 & 9.7 & 5.0 & 7.0 & 5.2 & 9.1 & 6.2 & 10.8 & 5.8 & 10.2 \\
\hline
\end{tabular}

${ }^{a} E D$, Emergency department; PCP, Primary care physician. 
Table 4 Adjusted effect of amitriptyline, duloxetine, and gabapentin, compared to pregabalin, on health care utilization during the follow-up period ${ }^{\mathrm{a}}$

\begin{tabular}{|c|c|c|c|}
\hline Health care utilization & Amitriptyline, RR $(95 \% \mathrm{CI})^{\mathrm{b}}$ & Duloxetine, RR $(95 \% \mathrm{CI})^{\mathrm{b}}$ & Gabapentin, RR $(95 \% \mathrm{Cl})^{\mathrm{b}}$ \\
\hline Outpatient visits $^{c}$ & $0.91(0.84 \text { to } 0.99)^{*}$ & $0.94(0.88 \text { to } 1.00)^{*}$ & $0.94(0.88 \text { to } 1.00)^{*}$ \\
\hline PCP visits ${ }^{c}$ & 0.99 (0.90 to 1.09$)$ & $0.92(0.85 \text { to } 0.98)^{*}$ & $0.92(0.85 \text { to } 0.98)^{*}$ \\
\hline Any specialist visits ${ }^{c}$ & $0.85(0.77 \text { to } 0.95)^{*}$ & $0.89(0.82 \text { to } 0.97)^{*}$ & $0.89(0.82 \text { to } 0.97)^{*}$ \\
\hline Prescription drugs ${ }^{c}$ & 0.97 (0.91 to 1.03$)$ & $0.94(0.90 \text { to } 0.98)^{*}$ & $0.94(0.90 \text { to } 0.98)^{*}$ \\
\hline Acute hospitalization $^{d}$ & $0.70(0.62 \text { to } 0.80)^{*}$ & $0.75(0.68 \text { to } 0.83)^{*}$ & 0.95 (0.89 to 1.01$)$ \\
\hline ED visits ${ }^{c}$ & 0.94 (0.86 to 1.03$)$ & $0.85(0.79 \text { to } 0.91)^{*}$ & 1.05 (1.00 to 1.10$)$ \\
\hline Physical therapy ${ }^{d}$ & $0.92(0.80$ to 1.06$)$ & $0.81(0.71 \text { to } 0.92)^{*}$ & $1.12(1.03$ to 1.21$)$ \\
\hline
\end{tabular}

${ }^{a} \mathrm{Cl}$, Confidence interval; ED, Emergency department; PCP, Primary care physician; RR, Rate ratio. ${ }^{\mathrm{b}}$ Pregabalin was used as the reference group. ${ }^{\mathrm{c}}$ Multivariable Poisson regression was used. ${ }^{d}$ Multivariable Cox proportional hazards regression was used. * Statistically significant difference $(P<0.05)$. All rate ratios were adjusted for neuropathic pain and the number of outpatient visits, PCP visits, any specialist visits, prescription drugs, acute hospitalizations, and ED visits at baseline.

fibromyalgia, including patient education, exercise, and mental health services, with the goal of reducing health care utilization.

There are limitations to our study. First, selection of a drug was not random in this observational cohort. We used rigorous pharmacoepidemiologic methods in the study design and data analysis, such as the new user design and PS-matched analysis, to minimize confounding by indication $[19,31]$. However, confounding by unmeasured variables, such as severity or activity of fibromyalgia, functional status, other comorbidities, use of nonpharmacologic treatments, and socioeconomic status, can still be an issue. For example, differences existed in comorbidities prior to PS matching, such that duloxetine initiators had a higher proportion of diagnosis and treatment for depression $(24 \%)$ and anxiety (21\%) than the pregabalin group (12\% and $13 \%$, respectively) [15]. If the presence or the severity of such conditions, which are related to use of health care systems, were incompletely controlled for, this could lead to residual confounding in our analysis. Second, there was also potential for misclassification, as we relied on diagnosis codes and drug-dispensing records to select patients with fibromyalgia and identify their comorbidities. Third, in this study, the reasons for use of health care resources (for example, outpatient and ED visits, hospitalization) were not available. Fourth, some of the visits to physicians or EDs may not have been related to fibromyalgia. Furthermore, the number of physician visits may not be an appropriate indicator of the effectiveness of fibromyalgia treatment. Fifth, we did not directly compare health care utilization between amitriptyline, duloxetine, and gabapentin initiators. Sixth, although it is possible that pharmacologic treatment has a beneficial effect on health care utilization in patients with fibromyalgia compared to no treatment, our study was designed to examine the comparative effectiveness of the four study drugs. Seventh, because access to health care is different in each health insurance plan in the United States, our results may not be generalizable to populations covered by different health insurance plans, particularly in different countries. Eighth, we did not examine the effect of combination therapy, but less than $10 \%$ of patients were put on combination therapy during the study period [15].

\section{Conclusions}

Fibromyalgia patients had substantial health care utilization before and after initiation of amitriptyline, duloxetine, gabapentin, or pregabalin and generally had high

Table 5 Adjusted effect of amitriptyline, duloxetine, and gabapentin, compared to pregabalin, on health care utilization in patients with treatment duration of at least 180 days ${ }^{\mathrm{a}}$

\begin{tabular}{|c|c|c|c|}
\hline Health care utilization & Amitriptyline, RR $(95 \% \mathrm{Cl})^{\mathrm{b}}$ & Duloxetine, RR $(95 \% \mathrm{Cl})^{\mathrm{b}}$ & 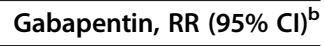 \\
\hline Outpatient visits $^{c}$ & $0.91(0.84 \text { to } 0.99)^{*}$ & $0.94(0.88 \text { to } 1.00)^{*}$ & $0.94(0.88 \text { to } 1.00)^{*}$ \\
\hline PCP visits ${ }^{c}$ & $0.99(0.90$ to 1.09$)$ & $0.92(0.85 \text { to } 0.98)^{*}$ & $0.92(0.85 \text { to } 0.98)^{*}$ \\
\hline Any specialist visits ${ }^{c}$ & $0.85(0.77 \text { to } 0.95)^{*}$ & $0.89(0.82 \text { to } 0.97)^{*}$ & $0.89(0.82 \text { to } 0.97)^{*}$ \\
\hline Prescription drugs ${ }^{c}$ & $0.97(0.91$ to 1.03$)$ & $0.94(0.90 \text { to } 0.98)^{*}$ & $0.94(0.90 \text { to } 0.98)^{*}$ \\
\hline Acute hospitalization $^{d}$ & $0.63(0.41 \text { to } 0.95)^{*}$ & $0.76(0.57$ to 1.01$)$ & $0.73(0.59 \text { to } 0.90)^{*}$ \\
\hline ED visits ${ }^{c}$ & 1.07 (0.84 to 1.37$)$ & 1.00 (0.83 to 1.19$)$ & 1.03 (0.90 to 1.17$)$ \\
\hline Physical therapy ${ }^{\mathrm{d}}$ & $0.76(0.55$ to 1.06$)$ & 0.85 (0.67 to 1.09$)$ & 0.99 (0.82 to 1.19$)$ \\
\hline
\end{tabular}

${ }^{\mathrm{a}} \mathrm{Cl}$, Confidence interval; ED, Emergency department; $\mathrm{PCP}$, Primary care physician; RR, Rate ratio. ${ }^{\mathrm{b}}$ Pregabalin was used as the reference group. ${ }^{\mathrm{C}} \mathrm{Multivariable}$ Poisson regression was used. ${ }^{d}$ Multivariable Cox proportional hazards regression was used. *Statistically significant difference $(P<0.05)$. All rate ratios were adjusted for neuropathic pain and the number of outpatient visits, PCP visits, any specialist visits, prescription drugs, acute hospitalizations, and ED visits at baseline. 
discontinuation rates of these medications. Fibromyalgia treatment did not substantially decrease health care utilization. Compared to pregabalin initiators, duloxetine initiators had less health care utilization during follow-up. In future studies, researchers should examine the effectiveness of pharmacologic treatments combined with nonpharmacologic interventions in treating chronic pain, as well as the use of health care resources in fibromyalgia.

\section{Additional files}

Additional file 1: Fibromyalgia related drugs. List of fibromyalgia-related drug names.

Additional file 2: Algorithms to define comorbidities. List of diagnosis and procedure codes to define comorbidities.

\begin{abstract}
Abbreviations
BZD: Benzodiazepine; Cl: Confidence interval; ED: Emergency department; FDA: US Food and Drug Administration; Gl: Gastrointestinal; ICD: International Classification of Diseases, Ninth Revision, Clinical Modification; NSAID: Nonsteroidal anti-inflammatory drug; PCP: Primary care physician; PDC: Proportion of days covered; PS: Propensity score; RR: Rate ratio; SD: Standard deviation; SNRI: Serotonin and noradrenaline reuptake inhibitor; SSRI: Selective serotonin reuptake inhibitor; TCA: Tricyclic antidepressant.
\end{abstract}

\section{Competing interests}

This study was supported by an investigator-initiated grant from Pfizer. Pfizer had no role in the study design, data collection, data analysis, or writing of the manuscript, nor did it have a role in approval of the content of the submitted manuscript. Publication of this article was not contingent on the approval of Pfizer. The study was conducted by the authors independently of the sponsor. The sponsor was given the opportunity to make nonbinding comments on a draft of the manuscript, but the authors retained the right of publication and to determine the final wording of the article. YCL received research grants from Forest. She holds stock in Cubist, Merck, Perrigo, and Express Scripts. SCK received a research grant from Forest. JEL has no competing interests. None of the authors had any nonfinancial competing interests related to this study.

\section{Authors' contributions}

SCK and YCL conceived of the study. SCK, JEL, and YCL participated in the study design and data interpretation. SCK and JEL performed data analysis. SCK drafted the manuscript. All authors read and approved the final manuscript.

\section{Acknowledgements}

This study was supported by an investigator-initiated grant from Pfizer. SCK is supported by National Institutes of Health (NIH) grant K23 AR059677 and a research grant from Pfizer. YCL is supported by NIH grants K23 AR57578 and R01 AR064850 and a research grant from Forest.

\section{Author details}

${ }^{1}$ Division of Pharmacoepidemiology and Pharmacoeconomics, Brigham and Women's Hospital and Harvard Medical School, 1620 Tremont St, Suite 3030, Boston, MA 02120, USA. ²Division of Rheumatology, Immunology and Allergy, Brigham and Women's Hospital and Harvard Medical School, 1620 Tremont St, Suite 3030, Boston, MA 02120, USA.

Received: 10 September 2014 Accepted: 19 January 2015

Published online: 28 January 2015

\section{References}

1. Wolfe F. The epidemiology of fibromyalgia. J Musculoskelet Pain. 1993;1:137-48

2. Wolfe F, Ross K, Anderson J, Russell I. Aspects of fibromyalgia in the general population: sex, pain threshold, and fibromyalgia symptoms. J Rheumatol. 1995;22:151-6.
3. Wolfe F, Clauw DJ, Fitzcharles MA, Goldenberg DL, Katz RS, Mease P, et al. The American College of Rheumatology preliminary diagnostic criteria for fibromyalgia and measurement of symptom severity. Arthritis Care Res (Hoboken). 2010;62:600-10.

4. Clauw D, Crofford L. Chronic widespread pain and fibromyalgia: what we know, and what we need to know. Best Pract Res Clin Rheumatol. 2003;17:685-701.

5. Hassett AL, Gevirtz RN. Nonpharmacologic treatment for fibromyalgia: patient education, cognitive-behavioral therapy, relaxation techniques, and complementary and alternative medicine. Rheum Dis Clin North Am. 2009;35:393-407.

6. Häuser W, Bernardy K, Uçeyler N, Sommer C. Treatment of fibromyalgia syndrome with gabapentin and pregabalin: a meta-analysis of randomized controlled trials. Pain. 2009;145:69-81.

7. Häuser W, Walitt B, Fitzcharles MA, Sommer C. Review of pharmacological therapies in fibromyalgia syndrome. Arthritis Res Ther. 2014;16:201.

8. White L, Robinson R, Yu A, Kaltenboeck A, Samuels S, Mallett D, et al. Comparison of health care use and costs in newly diagnosed and established patients with fibromyalgia. J Pain. 2009;10:976-83.

9. Robinson RL, Kroenke K, Williams DA, Mease P, Chen Y, Faries D, et al. Longitudinal observation of treatment patterns and outcomes for patients with fibromyalgia: 12-month findings from the reflections study. Pain Med. 2013;14:1400-15.

10. Gore M, Tai KS, Chandran A, Zlateva G, Leslie D. Clinical comorbidities, treatment patterns, and healthcare costs among patients with fibromyalgia newly prescribed pregabalin or duloxetine in usual care. J Med Econ. 2012;15:19-31.

11. Peng X, Sun P, Novick D, Andrews J, Sun S. Real-world comparison of health care utilization between duloxetine and pregabalin initiators with fibromyalgia. J Pain Res. 2014;7:37-46.

12. Sun $P$, Peng $X$, Sun $S$, Novick D, Faries DE, Andrews JS, et al. Direct medical costs and medication compliance among fibromyalgia patients: duloxetine initiators vs. pregabalin initiators. Pain Pract. 2014;14:22-31.

13. Zhao $Y$, Sun $P$, Watson P, Mitchell B, Swindle R. Comparison of medication adherence and healthcare costs between duloxetine and pregabalin initiators among patients with fibromyalgia. Pain Pract. 2011;11:204-16.

14. Gore M, Tai KS, Chandran A, Zlateva G, Leslie D. Clinical characteristics, pharmacotherapy, and healthcare resource use among patients with fibromyalgia newly prescribed pregabalin or tricyclic antidepressants. J Med Econ. 2012;15:32-44.

15. Kim SC, Landon JE, Solomon DH. Clinical characteristics and medication uses among fibromyalgia patients newly prescribed amitriptyline, duloxetine, gabapentin, or pregabalin. Arthritis Care Res (Hoboken). 2013;65:1813-9. doi:10.1002/acr.22071.

16. Gore M, Sadosky A, Zlateva G, Clauw D. Clinical characteristics, pharmacotherapy and healthcare resource use among patients with fibromyalgia newly prescribed gabapentin or pregabalin. Pain Pract. 2009;9:363-74

17. Deyo R, Cherkin D, Ciol M. Adapting a clinical comorbidity index for use with ICD-9-CM administrative databases. J Clin Epidemiol. 1992;45:613-9.

18. Schneeweiss S, Seeger J, Maclure M, Wang P, Avorn J, Glynn R. Performance of comorbidity scores to control for confounding in epidemiologic studies using claims data. Am J Epidemiol. 2001;154:854-64.

19. Rubin D. Estimating causal effects from large data sets using propensity scores. Ann Intern Med. 1997;127:757-63.

20. Parsons LS. Reducing bias in a propensity score matched-pair sample using greedy matching techniques. Sugi 26 Proceedings. Long Beach, CA; April 22-25, 2001 Cary, NC: SAS Institute; 2001. p. 214-26. http://www2.sas.com/ proceedings/sugi26/p214-26.pdf. Accessed 30 January 2015.

21. Cameron AC, Trivedi PK. Regression analysis of count data. Cambridge, UK: Cambridge University Press; 1998.

22. McCracken C, Dalgard OS, Ayuso-Mateos JL, Casey P, Wilkinson G, Lehtinen $\checkmark$, et al. Health service use by adults with depression: community survey in five European countries. Evidence from the ODIN study. Br J Psychiatry. 2006;189:161-7.

23. Wolfe F, Walitt BT, Katz RS, Lee YC, Michaud KD, Häuser W. Longitudinal patterns of analgesic and central acting drug use and associated effectiveness in fibromyalgia. Eur J Pain. 2013;17:581-6.

24. Häuser W, Jung E, Erbslöh-Möller B, Gesmann M, Kühn-Becker H, Petermann $F$, et al. The German fibromyalgia consumer reports-a cross-sectional survey. BMC Musculoskelet Disord. 2012;13:74. doi:10.1186/1471-2474-13-74. 
25. Nüesch $E$, Häuser W, Bernardy K, Barth J, Jüni P. Comparative efficacy of pharmacological and non-pharmacological interventions in fibromyalgia syndrome: network meta-analysis. Ann Rheum Dis. 2013;72:955-62.

26. Li YH, Wang FY, Feng CQ, Yang XF, Sun YH. Massage therapy for fibromyalgia: a systematic review and meta-analysis of randomized controlled trials. PLoS One. 2014;9:e89304.

27. Lima TB, Dias JM, Mazuquin BF, da Silva CT, Nogueira RM, Marques AP, et al. The effectiveness of aquatic physical therapy in the treatment of fibromyalgia: a systematic review with meta-analysis. Clin Rehabil. 2013;27:892-908

28. Naumann J, Sadaghiani C. Therapeutic benefit of balneotherapy and hydrotherapy in the management of fibromyalgia syndrome: a qualitative systematic review and meta-analysis of randomized controlled trials. Arthritis Res Ther. 2014;16:R141.

29. Häuser W, Klose P, Langhorst J, Moradi B, Steinbach M, Schiltenwolf M, et al Efficacy of different types of aerobic exercise in fibromyalgia syndrome: a systematic review and meta-analysis of randomised controlled trials. Arthritis Res Ther. 2010;12:R79.

30. Bernardy K, Klose P, Busch AJ, Choy EH, Häuser W. Cognitive behavioural therapies for fibromyalgia. Cochrane Database Syst Rev. 2013;9:CD009796.

31. Walker A. Confounding by indication. Epidemiology. 1996;7:335-6.

\section{Submit your next manuscript to BioMed Central and take full advantage of:}

- Convenient online submission

- Thorough peer review

- No space constraints or color figure charges

- Immediate publication on acceptance

- Inclusion in PubMed, CAS, Scopus and Google Scholar

- Research which is freely available for redistribution 